\title{
Féeries
}

Études sur le conte merveilleux, XVII $-\mathrm{XIX}{ }^{\mathrm{e}}$ siècle

\section{L'Invention d'un mythe : Psyché - Allégorie et fiction du siècle de Platon au temps de La Fontaine}

Véronique Gély, Champion, 2006, 557 p.

\section{Christelle Bahier-Porte}

\section{OpenEdition}

\section{Journals}

Édition électronique

URL : http://journals.openedition.org/feeries/433

DOI : $10.4000 /$ feeries.433

ISSN : 1957-7753

\section{Éditeur}

UGA Éditions/Université Grenoble Alpes

\section{Édition imprimée}

Date de publication : 1 octobre 2007

Pagination : $235-243$

ISBN : 978-2-84310-101-4

ISSN : 1766-2842

\section{Référence électronique}

Christelle Bahier-Porte, «L'Invention d'un mythe : Psyché - Allégorie et fiction du siècle de Platon au temps de La Fontaine », Féeries [En ligne], 4 | 2007, mis en ligne le 03 novembre 2008, consulté le 22 septembre 2020. URL : http://journals.openedition.org/feeries/433 ; DOI : https://doi.org/10.4000/ feeries. 433

Ce document a été généré automatiquement le 22 septembre 2020

(c) Féeries 


\title{
L'Invention d'un mythe : Psyché - Allégorie et fiction du siècle de Platon au temps de La Fontaine
}

Véronique Gély, Champion, 2006, 557 p.

\author{
Christelle Bahier-Porte
}

1 Cet ouvrage est le remaniement d'une thèse de littérature comparée soutenue en 1993 et se donne pour objet ambitieux l'étude de la création d'un mythe : comment naît en particulier un mythe littéraire ? Comment Psyché, l'âme et le papillon, accède-t-elle au statut de mythe au même titre que Pandore, Hercule, Prométhée ou Narcisse, autant de noms desquels Véronique Gély rapproche Psyché au cours de son étude.

2 Les enjeux théoriques d'un tel programme sont clairement définis dans l'introduction qui plaide, dès son titre "pour un mythe de Psyché » et s'ouvre sur l'avertissement suivant: «ce sont les temps modernes, c'est le christianisme qui a fait de Psyché un mythe » (p. 13). En effet, si l'on prend pour point de départ de l'étude de Psyché, le récit d'Apulée dans ses Métamorphoses, le statut de mythe de Psyché ne va pas de soi. Véronique Gély rappelle alors, et c'est un des postulats essentiels de son livre, que Psyché ne naît pas avec Apulée mais qu'il existe toute une tradition antique, iconographique, philosophique ou littéraire autour du nom de Psyché et de son interprétation. Le récit d'Apulée n'est donc en aucun cas un "texte fondateur ", d'ailleurs V.Gély refuse l'idée de rechercher un quelconque texte qui fonderait le mythe de Psyché et propose une approche "phénoménologique » : définir le mythe de Psyché à partir de ses multiples manifestations dans les arts, la philosophie, la littérature antérieurs au récit d'Apulée d'une part et d'autre part en rapport avec la réception du texte d'Apulée du Moyen-Âge à la Renaissance. Le mythe de Psyché se serait donc construit, à partir d'images parfois contradictoires, à travers les siècles, et ne devient un «mythologisme » recensé dans les dictionnaires qu'au XvII ${ }^{\mathrm{e}}$ siècle.

3 La perspective adoptée n'est pas seulement chronologique et une des problématiques de l'ouvrage repose sur le rapport entre allégorie et mythe. Psyché est une figure intéressante pour étudier la naissance d'un mythe tel que l'explique l'auteur, car elle se 
situe à la jonction du paganisme antique et du christianisme, elle est "d'emblée indissociable de la pensée allégorique et de l'expression allégorique » (p. 19). Le mythe de Psyché naîtrait d'abord de l'allégorie, plus précisément de la rencontre entre allégorie et conte, par le biais du texte d'Apulée. En dernier ressort, le texte d'Apulée est bien fondateur, non pas de la figure de Psyché, qui connaissait déjà de multiples interprétations mais bien de ce passage entre l'allégorie et le mythe qui se nourrit de la plurivocité. Contrairement à une idée reçue, le mythe naît ici du conte.

tes étudié s'arrête au début des années 1670 avec Les Amours de Psyché et Cupidon de La Fontaine (1669) et la Pysché, tragédie-ballet de Molière, Corneille, Lulli et Quinault en 1671 qui marquent la fin d'un cycle d'évolution du mythe, les textes de Corneille Molière et La Fontaine devenant à leur tour sources de réécritures. Véronique Gély se propose alors d'étudier l'invention du mythe de Psyché «depuis les premières représentations figurées que nous avons conservées, jusqu'au siècle de Charles I, Philippe IV et Louis XIV » (p. 19). Le parcours est impressionnant autant dans le temps que dans l'espace: Véronique Gély étudie les Psyché antiques, grecques et latines, italiennes, espagnoles, anglaises ou encore portugaises. C'est bien une archéologie patiente, précise, érudite du mythe de Psyché qui nous est proposée, dans toutes ses variations. Pour cela, Véronique Gély cite de très nombreux textes, dans leur langue d'origine, accompagnés de traductions et offre des illustrations qui donnent à voir la fortune iconographique de Psyché depuis les sarcophages de l'Antiquité jusqu'aux estampes $\mathrm{du} \mathrm{xvII}{ }^{\mathrm{e}}$ siècle. Une très riche bibliographie primaire et secondaire ainsi qu'un index des noms propres permettent de trouver aisément les références de textes ou d'auteurs souvent inconnus.

L'ouvrage se compose de deux parties. La première traite de l'allégorie de Psyché en deux chapitres consacrés l'un aux "allégorèmes ", c'est-à-dire aux motifs récurrents associés à Psyché, à partir notamment des significations données à son nom ; l'autre aux "allégorèses » : c'est-à-dire aux interprétations de l'allégorie. La seconde partie montre ensuite que c'est à partir de la contestation du statut d'allégorie de Psyché, des humanistes à Perrault, que Psyché devient une «fable mythologique », et rencontre la problématique des genres. Les derniers chapitres retrouvent néanmoins la perspective herméneutique de la première partie, en montrant comment par les thèmes du regard et de la voix, l'histoire de Psyché autorise une réflexion esthétique.

Le mythe semble particulièrement attaché au nom propre, à la différence du conte qui repose plutôt sur une structure narrative dont les héros peuvent changer de nom au gré des adaptations. Or le nom de Psyché ne semble pas évoquer à lui seul un récit. Les manuels de mythologie des humanistes ne le mentionnent pas et les poètes l'utilisent fort peu comme métaphore (V. G n'a relevé que deux exemples). Il y aurait alors de la part des humanistes une "volonté délibérée de ne pas considérer [Psyché] comme un personnage de la Fable» (p. 30). Pourtant, elle occupe une place importante dans les Mythologies de Fulgence et La généalogie des dieux païens de Boccace (1347-1359) et se trouve au centre d'un texte fondamental de la Renaissance: Le Songe de Poliphile de Francesco Colonna (1499). Mais ces textes ne créent pas un mythe, ils sont d'abord essentiellement allégoriques. En effet, Psyché est d'abord "une image»: depuis l'Antiquité grecque elle est représentée par un papillon ou une femme papillon tout en étant la personnification d'une entité abstraite (l'âme). C'est d'abord ce rapport entre l'image et l'allégorie que Véronique Gély décrit dans le premier chapitre en affirmant 
que tant que Psyché reste attachée à un sens, elle est allégorie, mais que lorsque le récit " déborde de l'image », elle " entre dans l'univers du mythe littéraire » (p. 41).

7 La première partie de l'ouvrage après cette mise au point théorique propose donc un inventaire des lieux communs associés à Psyché. Psyché est d'abord, depuis Platon et Homère, l'âme, au sens de souffle vital (anima). Cette idée de souffle vital est alors idéalement représentée dans l'iconographie par le papillon ou la jeune femme ailée. L'âme-psyché a partie liée avec les éléments constitutifs de la vie : air, feu, eau que ce soit dans la philosophie pré-socratique ou dans la doctrine hermétique et c'est avec Platon que l'âme échappe à ces éléments naturels (p. 66). Aristote quant à lui décrit la naissance de l'âme en inventant le concept d'entelechie (une forme d'énergie agissante et efficace) qui sera personnifié par Martianus Capella pour devenir la mère de Psyché. Le rapport de Psyché aux éléments naturels la rapproche alors du mythe de Pandore, cette parenté sera étudiée sous différentes formes au cours de l'ouvrage, elle est ici rattachée au mythe de Prométhée à partir d'un texte de Plotin qui réunit les deux figures.

Avec Les Noces de Mercure et de Philologie de Martianus Capella, ouvrage du $\mathrm{v}^{\mathrm{e}}$ siècle auquel V. Gély donne une importance centrale pour l'évolution des images de Psyché, Psyché devient en tant que telle, objet de discours et non plus illustration de l'entrée dans la vie, ou dans la mort, de l'homme. Pour évoquer la naissance de Psyché, Capella reprend le motif folklorique du don des dieux. Chaque don est allégorique et les commentateurs ultérieurs du texte, lus et analysés par V. Gély en donnent des clefs. L'objet de Capella n'est cependant pas de mettre en récit l'histoire de Psyché mais de décrire les attributs de l'âme, de "montrer l'éternité de l'âme en faisant mine de raconter sa naissance »: le texte de Capella est allégorique au sens strict. V. Gély poursuit à cette occasion le parallèle avec la naissance de Pandore et en étudie la résurgence à travers les siècles et les nations dans la reprise du motif du jardin des délices (p.107-112). Psyché, est donc une nouvelle allégorie de l'âme comme l'est Pandore chez Plotin, elle reçoit le don des sens et par métonymie devient aussi un corps, ce qui permet la création d'un couple allégorique: les sens se trouvant personnifiés dans une figure masculine (p.113). Ce principe masculin sera successivement Hermès, Prométhée, puis Éros. Cette union entre Éros et Psyché sera ensuite célébrée par la poésie et consacrée par Platon et perdure bien après le conte d'Apulée. Véronique Gély montre alors comment elle a été interprétée dans une perspective chrétienne comme l'union de l'âme et de l'amour divin. Cette partie est assez exemplaire de la méthode de Véronique Gély qui se fonde sur des lectures très nombreuses avec un souci d'exhaustivité qui n'évite pas toujours l'écueil de l'inventaire. Ainsi, l'allégorie de l'union de l'âme et de l'amour divin se retrouve dans les emblèmes chrétiens de Vaenius, Hermann Hugo ou encore Pierre Joulet (Amours spirituels de Psiché, 1600), chez un poète allemand Johann Scheffler (1625-1677), en Angleterre, en Espagne, en France. Chacune des occurrences est citée et analysée sur près d'une vingtaine de pages.

9 Par contraste, la conclusion de ce vaste parcours des « images » de Psyché à travers les siècles et les nations, paraît un peu laconique mais souligne, à juste titre, la puissance poétique de ces images " passées en revue » qui ne peuvent se réduire aux « sources » d'un mythe auquel Apulée aurait donné forme mais persistent en tant qu'images et créent de nouvelles allégories. 
10 Le second chapitre passe alors de l'image au commentaire, en s'intéressant aux différentes "allégorèses» de la figure, ou du récit, de Psyché. Les interprétations philosophiques sont d'abord étudiées car « ce sont d'abord les philosophes qui se sont intéressés à [Psyché]»(p.151): Platon, Plotin, Fulgence puis Martianus Capella. L'allégorie chrétienne de Fulgence dont les Mythologies ont eu une grande influence jusqu'à la fin de la Renaissance. L'allégorie de Martianus Capella sur les attributs de l'âme est quant à elle abondamment commentée dès le $\mathrm{IX}^{\mathrm{e}}$ siècle. À partir du XII ${ }^{\mathrm{e}}$ siècle, ces différentes interprétations convergent et « une tradition allégorique de la fable de Psyché se constitue ", à partir de deux versions opposées : la rédemption de Psyché chez Apulée, la chute de l'âme humaine chez Capella. V. Gély étudie les synthèses de Bernard Silvestre puis de Boccace. La plupart des œuvres de la Renaissance suivront l'interprétation religieuse du mythe, celle de Fulgence ou de Boccace (p.164). La question de la vocation édifiante de ce mythe en constitution est ensuite posée dans les œuvres artistiques. V. G étudie alors les estampes et les auto-sacramentales espagnols. Calderon fait, par exemple, correspondre l'interdit de Cupidon et le mystère de l'Eucharistie et Psyché se retrouve également dans le théâtre d'édification religieuse en Angleterre, dans le genre du « masque » pratiqué par Thomas Heywood.

11 Mais Psyché n'est pas seulement une allégorie philosophique ou religieuse, elle est aussi un «mythe de cour ». Les grandes familles d'Italie, les Gonzague de Mantoue et les Médicis à Florence en font leur emblème politique. C'est l'association de Psyché avec Médicis qui expliquerait d'ailleurs l'entrée de Psyché aux cours de France et d'Angleterre (p. 201) par le biais du ballet de cour notamment.

Une dernière section du chapitre étudie encore les jeux de mots autour des noms de Psyché et d'Éros notamment chez les virtuoses espagnols du concetto. Intéressante en soi et illustrée de citations nombreuses, cette partie crée néanmoins une distorsion par rapport au reste du chapitre, on ne saisit pas très bien en quoi le jeu de mot, le proverbe ou le paradoxe relèvent de l'allégorèse et en quoi ils enrichissent la signification du mythe de Psyché.

13 La seconde partie de l'ouvrage, intitulée "Forma » est centrée sur l'étude du conte d'Apulée, et sa réception tant littéraire qu'esthétique.

14 V. Gély rappelle en introduction que la lecture allégorique du conte d'Apulée ne va pas de soi: si le texte en lui-même semble appeler une interprétation, sa narratrice la récuse. Le récit destiné à la jeune Charité est fait pour la distraire et la fable de Psyché est qualifiée de "conte de vieille"; de la même façon Lucius éprouve un plaisir purement esthétique au récit du conte auquel il refuse de donner un plus haut sens. Le texte d'Apulée programme ainsi trois lectures différentes : allégorique, « existentielle » (Psyché comme modèle de comportement), esthétique, celle de Lucius qui fait alors figure dans cette perspective d'" ancêtre » du Poliphile de La Fontaine en mettant en avant le plaisir du conte (p.269). V. Gély, souligne alors le rôle de La Fontaine dans l'évolution définitive du mythe : «La Fontaine a laïcisé une fable qui lui était parvenue entièrement chrétienne [...] en l'écrivant comme un conte de fées ou un divertissement galant, il lui a permis, enfin, d'entrer dans la mythologie des modernes» (p. 270). C'est l'époque de l'entrée de Psyché dans les dictionnaires de mythologie, et dans l' Encyplopédie, La Fontaine acquiert le même statut qu'Apulée et Fulgence en tant qu'auteur de Psyché (p. 275). V. Gély rappelle néanmoins à juste titre que Perrault est «le véritable responsable du changement d'identité littéraire de Psyché» lorsqu'il 
démontre l'amoralité de cette fable pourtant auréolée du prestige de Platon et de Fulgence (p. 275).

Les études qui suivent s'intéressent alors aux métamorphoses génériques du conte de vieille (anilis fabula) proposé par Apulée que les adaptateurs qualifient tantôt de "milésienne", tantôt de "contes merveilleux». Les partisans de la milésienne rapprochent alors le récit du roman d'amour grec, les adaptations italiennes relèvent dans cette perspective du genre de la novella. Le récit-cadre d'Apulée présente pourtant une narration orale qui veut plaire à son auditoire. La « Psyché » de Tristan L'Hermitte insérée dans Le Page disgracié se place dans ce sillage et marque l'entrée de Psyché dans la littérature galante : le narrateur veut plaire à son public féminin qui s'empresse de débattre sur l'amour à l'occasion du récit (p. 309). La Fontaine modifiera la perspective strictement galante (récit fait à de jeunes femmes) pour adresser la lecture de Psyché à des auditeurs masculins et mettre en question les pouvoirs de la littérature (p. 311). Les adaptations du conte dans le genre "merveilleux » (Boiardo, Basile, La Fontaine, $\mathrm{M}^{\text {me }}$ d'Aulnoy) sont ensuite mentionnées. Il est dommage que ces rencontres de Psyché avec la littérature ne bénéficient pas d'une conclusion qui mettrait en avant les modalités et les choix littéraires présidant à ces mises en écriture du mythe.

Les derniers chapitres reviennent à l'herméneutique. Véronique Gély propose tout d'abord une « lecture intuitive » des enseignements de la fable de Psyché, lecture plus personnelle qui n'hésite pas à avoir recours aux critiques modernes, notamment psychanalytiques. V.G. pose la question de l'exemplarité de la fable de Psyché en abordant tour à tour l'identité symbolique d'Éros et la signification du parcours de Psyché en se fondant sur une analyse des «tensions entre la vie et la mort et entre le masculin et le féminin " présentes dès le conte d'Apulée. V.G. montre alors par un biais plus interprétatif que Psyché a pris le relais de Pandore : la Psyché d'Apulée serait une «Pandore sauvée » car elle résout les conflits que Pandore faisait naître (p. 357). Elle n'oublie pas néanmoins la tradition satirique qui pointe les défauts de Psyché ni le registre héroïque en montrant comment Psyché, dans les épopées espagnoles en particulier devient héroïne d'une « épopée de la paix » (p. 382).

Les deux derniers chapitres proposent enfin une interprétation d'ordre esthétique de la fable de Psyché comme mise en question de "beauté et de la forme» (p. 386) par l'étude de deux sens privilégiés dans le récit: la vue et la voix. L'interdit de Cupidon pose la question de la distinction entre les " yeux de chair » et les " yeux de l'âme », et concerne donc l'imagination et l'illusion. Le récit d'Apulée sert ici de point d'appui : «le conte de Psyché dans le roman d'Apulée s'ouvre sur un manifeste esthétique, une réflexion sur la beauté » qui devient « accessible » par le biais de la milésienne (p. 392). V. Gély examine ensuite comment cette réflexion sur la beauté est reprise par La Fontaine, les auteurs italiens Udine et Marino. Le motif du regard amène logiquement une réflexion sur l'illusion, et la reprise du conte dans l'univers baroque du desengaño (p. 397 et suiv.), ou au théâtre lorsqu'il permet la mise en scène de l'illusion comique. Le dernier chapitre examine dans une perspective similaire les liens entre le récit de Psyché et la voix, la musique et la connaissance qu'elles permettent d'acquérir. La présence de la musique est d'abord étudiée dans le conte d'Apulée qui explique peutêtre la «métamorphose » qui fait de Psyché au XVII siècle, une héroïne de pastorale romanesque ou dramatique mais le rapprochement avec la pastorale ou l'idylle vient moins directement du récit d'Apulée que de réminiscences ovidiennes (p.460) présentes chez Apulée mais surtout chez les poètes de la Renaissance ou encore chez La 
Fontaine. Elle montre ensuite comment certains adaptateurs (Heywood, Gabrielli) donnent à l'histoire de Psyché un cadre arcadien et termine son parcours par l'entrée de Psyché à l'opéra par le biais du genre de la pastorale et de la tragicomédie. Finalement, Psyché serait le «Conte des contes » car elle « est comme une genèse des genres littéraires : les motifs du conte donnent naissance à un art poétique. L'illusion engendre le théâtre, le monstre la tragicomédie, l'imagination le poème et le roman, la quête le poème épique » et réciproquement, les genres qui accueillent Psyché modifient les données de l'intrigue ou les personnages (p. 490).

Autant dire que c'est la littérature qui en se penchant sur le berceau de Psyché, favorisé des dieux, lui a donné accès au mythe. Les actes d'un colloque récent, intitulés précisément Les Métamorphoses de Psyché ${ }^{1}$, montrent également cette rencontre de Psyché et des arts du spectacle, de la scène à la peinture, comme si finalement Psyché était bien un mythe qui met d'abord en question notre manière de voir.

\section{NOTES}

1. Barbafieri (Carine), Rauseo (Chris) (dir.), Les Métamorphoses de Psyché, Presses universitaires de Valenciennes, 2004. 